\title{
Chemical Imaging with Auger Electron Spectroscopy
}

\author{
S.A. Wight
}

National Institute of Standards and Technology, Gaithersburg MD 20899-8371.

Auger electron spectroscopy (AES) is related to x-ray spectroscopy in that both are the result of a primary electron beam interacting with a specimen. A core electron is knocked out and as a higher energy level electron moves down to fill the core level vacancy, energy is released in the form of an Auger electron with the energy corresponding to the difference between the two levels. This is the basis for Auger electron spectroscopy (AES). Alternatively, the energy can be given off in the form of a characteristic $\mathrm{x}$-ray and this is the basis of $\mathrm{x}$-ray microanalysis.

Auger electrons are produced throughout a sample volume defined by the interaction of the primary electron beam and the specimen. Auger electrons are relatively low in energy and so can only travel a small distance in a solid. Only the Auger electrons that are created close to the surface, within a few nanometers, have sufficient mean free path to escape the specimen and be collected for analysis. Auger electrons are recorded as a function of their energy by the electron spectrometer in the Auger microscope and provide elemental as well as bonding information.

The surface sensitivity of AES requires the specimen to have a clean surface, free of contamination. For this reason, Auger microscopes are ultra high vacuum (UHV) in the specimen chamber, which is on the order of $10^{-8} \mathrm{~Pa}$. Steps must be taken to clean specimens prior to introduction into the Auger microscope so that they are free of volatile organic compounds that can contaminate the chamber vacuum. The Auger specimen chamber is equipped with an argon ion gun for sputter cleaning off the contamination or oxide layer that coats specimens as a result of transporting them in air.

Investigation of a buried structure or an interface that is deeper than the Auger escape depth can be accomplished by Auger depth profiling. In Auger depth profiling, the instrument alternates between Ar ion sputtering of the surface and Auger analysis of the surface until as material is sputtered away, the elemental composition changes with depth.

Auger and x-ray microanalysis are complimentary in that they can both provide elemental information from the specimen, however the depth of the information is different. X-ray analysis is microanalysis in that it collects information from the first few micrometers of the specimen. Auger analysis is nanoanalysis in that it represents information from the first few nanometers of the specimen. These two pieces of information can be used in a relative sense to view the composition of the bulk and the surface of a specimen simultaneously. Reducing the primary beam voltage can reduce the depth of the interaction area from which $\mathrm{x}$-rays are produced but this also reduces the number of x-ray lines available for analysis.

Either Auger or x-ray analysis can be applied by spotting the beam down on the specimen and collecting the spectrum. It is often desirable to collect a chemical composition image by collecting or monitoring the spectrometer response while scanning the electron beam over the specimen. There is a major difference in the way Auger and x-ray images are collected. Because of the simultaneous nature of energy dispersive x-ray data collection, the entire spectrum can be collected at each pixel 
and post processed for composition. However the Auger spectrometer is held at a particular energy for the entire chemical image data collection then moved to the next energy for the collection of the next element of interest. This procedure requires the choice of element and energy before the start of data collection and therefore post processing is not an option.

Demonstration of Auger and x-ray mapping or chemical compositional imaging of specimens are demonstrated. Figure 1 is an example of Auger mapping on an ion etched crater at the edge of an oxidation trench in a GaAs/AlGaAs multilayer.

\section{References}

[1] D. Briggs and J.T. Grant, Eds., Surface Analysis by Auger and X-Ray Photoelectron Spectroscopy, The Cromwell Press, Trowbridge UK, 2003.

[2] D. Briggs and M.P. Seah, Eds., Practical Surface Analysis, $2^{\text {nd }}$ Ed, Vol. 1: Auger and X-Ray Photoelectron Spectroscopy, John Wiley and Sons, West Sussex, England, 1983.

[3] J.F. Watts and J. Wolstenholme, An Introduction to Surface Analysis by XPS and AES, John Wiley and Sons, West Sussex, England, 2003.

[4] Certain commercial equipment, instruments, or materials are identified in this report to specify adequately the experimental procedure. Such identification does not imply recommendation or endorsement by the National Institute of Standards and Technology, nor does it imply that the materials or equipment identified are necessarily the best available for the purpose.
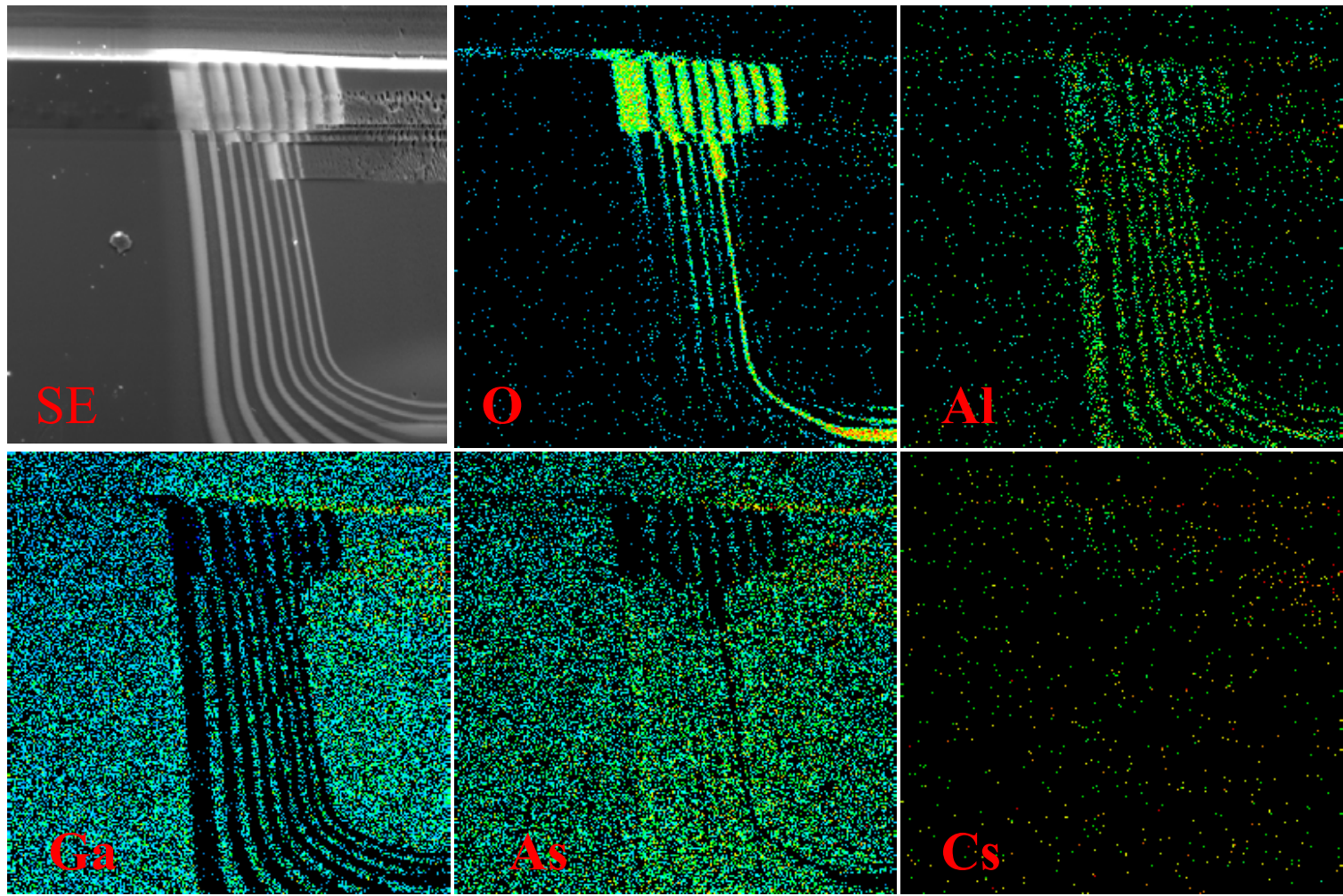

FIG. 1. Secondary electron image and Auger images (oxygen, aluminum, gallium, arsenic, and cesium) of a secondary ion mass spectrometry (SIMS) crater in the vicinity of the oxidation trench in a GaAs/AlGaAs multilayer. After sputtering the surface with low energy Ar ions to remove both atmospheric oxygen and cesium from the SIMS sputtering. 